\title{
A MOLP Method for Solving Fully Fuzzy Linear Programming with LR Fuzzy Parameters
}

\author{
Xiao-Peng Yang, ${ }^{1,2}$ Xue-Gang Zhou, ${ }^{1,3}$ Bing-Yuan Cao, ${ }^{1}$ and S. H. Nasseri ${ }^{4}$ \\ ${ }^{1}$ School of Mathematics and Information Science, Key Laboratory of Mathematics and Interdisciplinary Sciences of Guangdong, \\ Higher Education Institutes, Guangzhou University, Guangzhou 510006, China \\ ${ }^{2}$ Department of Mathematics and Statistics, Hanshan Normal University, Chaozhou 521041, China \\ ${ }^{3}$ Department of Applied Mathematics, Guangdong University of Finance, Guangzhou 510521, China \\ ${ }^{4}$ Department of Mathematics, Mazandaran University, Babolsar 47416-95447, Iran
}

Correspondence should be addressed to Bing-Yuan Cao; caobingy@163.com

Received 22 March 2014; Accepted 15 September 2014; Published 29 September 2014

Academic Editor: Yang Xu

Copyright (C) 2014 Xiao-Peng Yang et al. This is an open access article distributed under the Creative Commons Attribution License, which permits unrestricted use, distribution, and reproduction in any medium, provided the original work is properly cited.

Kaur and Kumar, 2013, use Mehar's method to solve a kind of fully fuzzy linear programming (FFLP) problems with LR fuzzy parameters. In this paper, a new kind of FFLP problems is introduced with a solution method proposed. The FFLP is converted into a multiobjective linear programming (MOLP) according to the order relation for comparing the LR flat fuzzy numbers. Besides, the classical fuzzy programming method is modified and then used to solve the MOLP problem. Based on the compromised optimal solution to the MOLP problem, the compromised optimal solution to the FFLP problem is obtained. At last, a numerical example is given to illustrate the feasibility of the proposed method.

\section{Introduction}

The research on fuzzy linear programming (FLP) has risen highly since Bellman and Zadeh [1] proposed the concept of decision making in fuzzy environment. The FLP problem is said to be a fully fuzzy linear programming (FFLP) problem if all the parameters and variables are considered as fuzzy numbers. In recent years, some researchers such as Lofti and Kumar were interested in the FFLP problems, and some solution methods have been obtained to the fully fuzzy systems [2-4] and the FFLP problems [5-13]. FFLP problems can be divided in two categories: (1) FFLP problems with inequality constraints; (2) FFLP problems with equality constraints. If the FFLP problems are classified by the types of the fuzzy numbers, they will include the next three classes: (1) FFLP problems with all the parameters and variables represented by triangular fuzzy numbers; (2) FFLP problems with all the parameters and variables represented by trapezoidal fuzzy numbers; (3) FFLP problems with all the parameters and variables expressed by $L R$ fuzzy numbers (or $L R$ flat fuzzy numbers).

Fuzzy programming method is a classical method to solve multiobjective linear programming (MOLP) $[14,15]$. In this paper, the fuzzy programming method is modified and then used to obtain a compromised optimal solution of the MOLP. The modified fuzzy programming method is shown in Steps 4-10 of the proposed method in Section 3.

Dehghan et al. [2-4] employed several methods to find solutions of the fully fuzzy linear systems. Hosseinzadeh Lotfi et al. [6] used the lexicography method to obtain the fuzzy approximate solutions of the FFLP problems. Allahviranloo et al. [7] and Kumar et al. [5, 8] solved the FFLP problem by use of a ranking function.

Fan et al. [12] adopted the $\alpha$-cut level to deal with a generalized fuzzy linear programming (GFLP) probelm. The feasibility of fuzzy solutions to the GFLP was investigated and a stepwise interactive algorithm based on the idea of design of experiment was advanced to solve the GFLP problem. 
Kaur and Kumar [9] introduced Mehar's method to the FFLP problems with $L R$ fuzzy parameters. They consider the following model:

Maximize (or Minimize)

$$
\sum_{j=1}^{n}\left(\left(p_{j}, q_{j}, \alpha_{j}^{\prime}, \beta_{j}^{\prime}\right)_{L R} \odot\left(x_{j}, y_{j}, \alpha_{j}^{\prime \prime}, \beta_{j}^{\prime \prime}\right)_{L R}\right)
$$

subject to

$$
\begin{array}{r}
\sum_{j=1}^{n}\left(\left(a_{i j}, b_{i j}, \alpha_{i j}, \beta_{i j}\right)_{L R}\right. \\
\left.\odot\left(x_{j}, y_{j}, \alpha_{j}^{\prime \prime}, \beta_{j}^{\prime \prime}\right)_{L R}\right) \preceq, \approx, \geq \\
\left(b_{i}, g_{i}, \gamma_{i}, \delta_{i}\right)_{L R}, \\
i=1,2, \ldots, m,
\end{array}
$$

where the parameters and variables are $L R$ flat fuzzy numbers and the order relation for comparing the numbers is defined as follows.

(i) $\widetilde{u} \preceq \widetilde{v}$ if and only if $\mathfrak{R}(\widetilde{u}) \leq \Re(\widetilde{v})$,

(ii) $\widetilde{u} \geq \widetilde{v}$ if and only if $\Re(\widetilde{u}) \geq \Re(\widetilde{v})$,

(iii) $\widetilde{u} \approx \widetilde{v}$ if and only if $\mathfrak{R}(\widetilde{u})=\Re(\widetilde{v})$.

Here $\tilde{u}$ and $\widetilde{v}$ are two arbitrary $L R$ flat fuzzy numbers.

In our study, we consider a new kind of FFLP problems with $L R$ flat fuzzy parameters as follows:

$\min ($ or $\max )$

$$
z(\tilde{x})=\widetilde{c}_{1} \otimes \tilde{x}_{1} \oplus \widetilde{c}_{2} \otimes \tilde{x}_{2} \oplus \cdots \oplus \widetilde{c}_{n} \otimes \tilde{x}_{n}
$$

subject to

$$
\begin{aligned}
& \tilde{a}_{i 1} \otimes \tilde{x}_{1} \oplus \tilde{a}_{i 2} \otimes \tilde{x}_{2} \oplus \cdots \oplus \tilde{a}_{i n} \otimes \tilde{x}_{n} \leq \tilde{b}_{i}, \\
& i=1,2, \ldots, m, \\
& \tilde{x}_{j} \geq \tilde{0}, \quad j=1,2, \ldots, n,
\end{aligned}
$$

where the parameters and variables are $L R$ flat fuzzy numbers and the order relation shown in Definition 4 is different from the one above.

In this paper, we modify the classical fuzzy programming method. The FFLP is changed into a MOLP problem solved by the modified fuzzy programming method. We get the compromised optimal solution to the MOLP and generate the corresponding compromised optimal solution to the FFLP.

The rest of the paper is organized as follows. In Section 2, the basic definitions and the FFLP model are introduced. In Section 3, we propose a MOLP method to solve the FFLP problems. Some results are discussed from the solutions obtained by the proposed method. In Section 4, a numerical example is given to illustrate the feasibility of the proposed method. In Section 5, we show some short concluding remarks.

\section{Preliminaries}

\subsection{Basic Notations}

Definition 1 (LR fuzzy number, see [2]). A fuzzy number $\tilde{u}$ is said to be an $L R$ fuzzy number if

$$
\widetilde{u}(x)=\left\{\begin{array}{lll}
L\left(\frac{m-x}{\alpha}\right), & x \leq m, & \alpha>0, \\
R\left(\frac{x-m}{\beta}\right), & x \geq m, \quad \beta>0,
\end{array}\right.
$$

where $m$ is the mean value of $\widetilde{u}$ and $\alpha$ and $\beta$ are left and right spreads, respectively, and function $L(\cdot)$ means the left shape function satisfying

(1) $L(x)=L(-x)$;

(2) $L(0)=1$ and $L(1)=0$;

(3) $L(x)$ is nonincreasing on $[0, \infty)$.

Naturally, a right shape function $R(\cdot)$ is similarly defined as $L(\cdot)$.

Definition 2 ( $L R$ flat fuzzy number, see $[9,16])$. A fuzzy number $\tilde{u}$, denoted as $(m, n, \alpha, \beta)_{L R}$, is said to be an $L R$ flat fuzzy number if its membership function $\widetilde{u}(x)$ is given by

$$
\widetilde{u}(x)= \begin{cases}L\left(\frac{m-x}{\alpha}\right), & x \leq m, \alpha>0, \\ R\left(\frac{x-n}{\beta}\right), & x \geq n, \beta>0, \\ 1, & m \leq x \leq n .\end{cases}
$$

Definition 3 (see $[5,9]$ ). An $L R$ flat fuzzy number $\tilde{u}=$ $(m, n, \alpha, \beta)_{L R}$ is said to be nonnegative $L R$ flat fuzzy number if $m-\alpha \geq 0$ and is said to be nonpositive $L R$ flat number if $n+\beta \leq 0$.

We define $\tilde{u}=(m, n, 0,0)_{L R}$ as an $L R$ fuzzy number with membership function

$$
\tilde{u}(x)= \begin{cases}1, & m \leq x \leq n \\ 0, & \text { otherwise }\end{cases}
$$

and denote $(0,0,0,0)_{L R}$ as $\widetilde{0}$. 
2.2. Arithmetic Operations. Let $\tilde{u}=\left(m_{1}, n_{1}, \alpha_{1}, \beta_{1}\right)_{L R}$ and $\widetilde{v}=$ $\left(m_{2}, n_{2}, \alpha_{2}, \beta_{2}\right)_{L R}$ be two $L R$ flat fuzzy numbers, $k \in R$. Then the arithmetic operations are given as follows $[9,16]$ :

$$
\begin{aligned}
& \tilde{u} \oplus \widetilde{v}=\left(m_{1}+m_{2}, n_{1}+n_{2}, \alpha_{1}+\alpha_{2}, \beta_{1}+\beta_{2}\right)_{L R}, \\
& \tilde{u} \ominus \widetilde{v}=\left(m_{1}-n_{2}, n_{1}-m_{2}, \alpha_{1}+\beta_{2}, \alpha_{2}+\beta_{1}\right)_{L R} \text {, } \\
& k \tilde{u}= \begin{cases}\left(k m_{1}, k n_{1}, k \alpha_{1}, k \beta_{1}\right)_{L R}, & k \geq 0, \\
\left(k n_{1}, k m_{1},-k \beta_{1},-k \alpha_{1}\right)_{R L}, & k<0,\end{cases} \\
& \tilde{u} \otimes \widetilde{v}=\left\{\begin{array}{r}
\left(m_{1} m_{2}, n_{1} n_{2}, m_{1} \alpha_{2}+\alpha_{1} m_{2}, n_{1} \beta_{2}+\beta_{1} n_{2}\right)_{L R}, \\
\tilde{u} \geq \widetilde{0}, \widetilde{v} \geq \widetilde{0}, \\
\left(m_{1} n_{2}, n_{1} m_{2}, \alpha_{1} n_{2}-m_{1} \beta_{2}, \beta_{1} m_{2}-n_{1} \alpha_{2}\right)_{L R}, \\
\tilde{u} \leq \widetilde{0}, \widetilde{v} \geq \widetilde{0}, \\
\left(n_{1} m_{2}, m_{1} n_{2}, n_{1} \alpha_{2}-\beta_{1} m_{2}, m_{1} \beta_{2}-\alpha_{1} n_{2}\right)_{L R}, \\
\widetilde{u} \geq \widetilde{0}, \widetilde{v} \leq \widetilde{0}, \\
\left(n_{1} n_{2}, m_{1} m_{2},-n_{1} \beta_{2}-\beta_{1} n_{2},-m_{1} \alpha_{2}-\alpha_{1} m_{2}\right)_{L R}, \\
\widetilde{u} \leq \widetilde{0}, \widetilde{v} \leq \widetilde{0} .
\end{array}\right.
\end{aligned}
$$

It is easy to verify that the operator $\oplus$ satisfies associative law. Hence, the formula $\sum_{j=1}^{n} \widetilde{u}_{j}=\widetilde{u}_{1} \oplus \widetilde{u}_{2} \oplus \cdots \oplus \tilde{u}_{n}$ is reasonable, where $\tilde{u}_{1}, \tilde{u}_{2}, \ldots, \tilde{u}_{n}$ are $L R$ flat fuzzy numbers.

2.3. Order Relation for Comparing the LR Flat Fuzzy Numbers. For comparing the $L R$ flat fuzzy numbers, we introduce the order relation as follows.

Definition 4. Let $\widetilde{u}=\left(m_{1}, n_{1}, \alpha_{1}, \beta_{1}\right)_{L R}$ and $\widetilde{v}=\left(m_{2}, n_{2}, \alpha_{2}\right.$, $\left.\beta_{2}\right)_{L R}$ be any $L R$ flat fuzzy numbers. Then

(i) $\tilde{u}=\widetilde{v}$ if and only if $m_{1}=m_{2}, n_{1}=n_{2}, \alpha_{1}=\alpha_{2}$, $\beta_{1}=\beta_{2}$

(ii) $\tilde{u} \leq \widetilde{v}$ if and only if $m_{1} \leq m_{2}, n_{1} \leq n_{2}, m_{1}-\alpha_{1} \leq$ $m_{2}-\alpha_{2}, n_{1}+\beta_{1} \leq n_{2}+\beta_{2}$

(iii) $\tilde{u} \geq \widetilde{v}$ if and only if $m_{1} \geq m_{2}, n_{1} \geq n_{2}, m_{1}-\alpha_{1} \geq$ $m_{2}-\alpha_{2}, n_{1}+\beta_{1} \geq n_{2}+\beta_{2}$.

Based on the definition of order $\leq$, we may obtain that (i) $\tilde{u}$ is nonnegative if and only if $\widetilde{u} \geq \widetilde{0}$; (ii) $\tilde{u}$ is nonpositive if and only if $\widetilde{u} \leq \widetilde{0}$.

The following propositions are given to show the properties of the order relation defined above.

Proposition 5. Let $\widetilde{u}, \widetilde{v}, \widetilde{w}, \widetilde{\sigma}$ be four arbitrary LR flat fuzzy numbers and $k$ an arbitrary real number. Then

(1) $\tilde{u} \leq \widetilde{v}, \quad \widetilde{w} \leq \widetilde{\sigma} \Longrightarrow \widetilde{u} \oplus \widetilde{w} \leq \widetilde{v} \oplus \widetilde{\sigma}$;

(2) $\tilde{u} \leq \widetilde{v} \Longrightarrow \begin{cases}k \tilde{u} \leq k \widetilde{v}, & k \geq 0, \\ k \tilde{u} \geq k \widetilde{v}, & k \leq 0 .\end{cases}$

Proof. Suppose $\widetilde{u}=\left(m_{1}, n_{1}, \alpha_{1}, \beta_{1}\right)_{L R}, \widetilde{v}=\left(m_{2}, n_{2}, \alpha_{2}, \beta_{2}\right)_{L R}$, $\widetilde{w}=\left(m_{3}, n_{3}, \alpha_{3}, \beta_{3}\right)_{L R}$, and $\widetilde{\sigma}=\left(m_{4}, n_{4}, \alpha_{4}, \beta_{4}\right)_{L R}$.
(1) It is obvious that $\tilde{u} \oplus \widetilde{w}=\left(m_{1}+m_{3}, n_{1}+n_{3}, \alpha_{1}+\alpha_{3}, \beta_{1}+\right.$ $\left.\beta_{3}\right)_{L R}, \widetilde{v} \oplus \widetilde{\sigma}=\left(m_{2}+m_{4}, n_{2}+n_{4}, \alpha_{2}+\alpha_{4}, \beta_{2}+\beta_{4}\right)_{L R}$. Since $\widetilde{u} \leq \widetilde{v}, \widetilde{w} \leq \widetilde{\sigma}$, we get

$$
\begin{array}{ll}
m_{1} \leq m_{2}, & n_{1} \leq n_{2}, \quad m_{1}-\alpha_{1} \leq m_{2}-\alpha_{2}, \\
& n_{1}+\beta_{1} \leq n_{2}+\beta_{2}, \\
m_{3} \leq m_{4}, & n_{3} \leq n_{4}, \quad m_{3}-\alpha_{3} \leq m_{4}-\alpha_{4}, \\
& n_{3}+\beta_{3} \leq n_{4}+\beta_{4} .
\end{array}
$$

So

$$
\begin{aligned}
m_{1}+m_{3} & \leq m_{2}+m_{4}, \\
n_{1}+n_{3} & \leq n_{2}+n_{4}, \\
\left(m_{1}+m_{3}\right)-\left(\alpha_{1}+\alpha_{3}\right) & \leq\left(m_{2}+m_{4}\right)-\left(\alpha_{2}+\alpha_{4}\right), \\
\left(n_{1}+n_{3}\right)+\left(\beta_{1}+\beta_{3}\right) & \leq\left(n_{2}+n_{4}\right)-\left(\beta_{2}+\beta_{4}\right) .
\end{aligned}
$$

This indicates that $\widetilde{u} \oplus \widetilde{w} \leq \widetilde{v} \oplus \widetilde{\sigma}$.

(2) It is clear that $k \tilde{u}=\left(k m_{1}, k n_{1}, k \alpha_{1}, k \beta_{1}\right)_{L R}, k \tilde{v}=$ $\left(k m_{2}, k n_{2}, k \alpha_{2}, k \beta_{2}\right)_{L R}$. From $\tilde{u} \leq \widetilde{v}$, we get

$$
\begin{array}{ll}
m_{1} \leq m_{2}, \quad n_{1} \leq n_{2}, \quad m_{1}-\alpha_{1} \leq m_{2}-\alpha_{2}, \\
& n_{1}+\beta_{1} \leq n_{2}+\beta_{2} .
\end{array}
$$

Therefore,

$$
\begin{array}{ll}
k m_{1} \leq k m_{2}, & k n_{1} \leq k n_{2}, \quad k m_{1}-k \alpha_{1} \leq k m_{2}-k \alpha_{2}, \\
& k n_{1}+k \beta_{1} \leq k n_{2}+k \beta_{2},
\end{array}
$$

for $k \geq 0$, and

$$
\begin{array}{ll}
k m_{1} \geq k m_{2}, \quad & k n_{1} \geq k n_{2}, \quad k m_{1}-k \alpha_{1} \geq k m_{2}-k \alpha_{2}, \\
& k n_{1}+k \beta_{1} \geq k n_{2}+k \beta_{2},
\end{array}
$$

for $k \leq 0$. This indicates that

$$
\begin{array}{ll}
k \widetilde{u} \leq k \widetilde{v}, & k \geq 0, \\
k \tilde{u} \geq k \widetilde{v}, \quad k \leq 0 .
\end{array}
$$

Proposition 6. Let $\widetilde{u}, \widetilde{v}, \widetilde{w}$ be three arbitrary LR flat fuzzy numbers. Then

(1) $\tilde{u} \leq \tilde{u}$;

(2) $\tilde{u} \leq \widetilde{v}, \widetilde{v} \leq \widetilde{u} \Rightarrow \widetilde{u}=\widetilde{v}$;

(3) $\tilde{u} \leq \widetilde{v}, \widetilde{v} \leq \widetilde{w} \Rightarrow \widetilde{u} \leq \widetilde{w}$.

Proof. Suppose $\tilde{u}=\left(m_{1}, n_{1}, \alpha_{1}, \beta_{1}\right)_{L R}, \widetilde{v}=\left(m_{2}, n_{2}, \alpha_{2}, \beta_{2}\right)_{L R}$, and $\widetilde{w}=\left(m_{3}, n_{3}, \alpha_{3}, \beta_{3}\right)_{L R}$.

(1) Obviously, $\widetilde{u}=\widetilde{u}$; hence, we have $\widetilde{u} \leq \widetilde{u}$. 
(2) Since $\tilde{u} \leq \widetilde{v}, \widetilde{v} \leq \widetilde{u}$, we get

$$
\begin{array}{ll}
m_{1} \leq m_{2}, & n_{1} \leq n_{2}, \quad m_{1}-\alpha_{1} \leq m_{2}-\alpha_{2}, \\
& n_{1}+\beta_{1} \leq n_{2}+\beta_{2}, \\
m_{2} \leq m_{1}, & n_{2} \leq n_{1}, \quad m_{2}-\alpha_{2} \leq m_{1}-\alpha_{1}, \\
& n_{2}+\beta_{2} \leq n_{1}+\beta_{1} .
\end{array}
$$

This means

$$
\begin{array}{ll}
m_{1}=m_{2}, \quad n_{1}=n_{2}, \quad m_{1}-\alpha_{1}=m_{2}-\alpha_{2}, \\
& n_{1}+\beta_{1}=n_{2}+\beta_{2} .
\end{array}
$$

That is

$$
m_{1}=m_{2}, \quad n_{1}=n_{2}, \quad \alpha_{1}=\alpha_{2}, \quad \beta_{1}=\beta_{2} .
$$

Therefore, we have $\widetilde{u}=\widetilde{v}$.

(3) From $\tilde{u} \leq \widetilde{v}, \widetilde{v} \leq \widetilde{w}$, we get

$$
\begin{array}{ll}
m_{1} \leq m_{2}, & n_{1} \leq n_{2}, \quad m_{1}-\alpha_{1} \leq m_{2}-\alpha_{2}, \\
& n_{1}+\beta_{1} \leq n_{2}+\beta_{2}, \\
m_{2} \leq m_{3}, & n_{2} \leq n_{3}, \quad m_{2}-\alpha_{2} \leq m_{3}-\alpha_{3}, \\
& n_{2}+\beta_{2} \leq n_{3}+\beta_{3} .
\end{array}
$$

This indicates

$$
\begin{array}{ll}
m_{1} \leq m_{3}, \quad n_{1} \leq n_{3}, \quad m_{1}-\alpha_{1} \leq m_{3}-\alpha_{3}, \\
& n_{1}+\beta_{1} \leq n_{3}+\beta_{3} .
\end{array}
$$

Therefore, we have $\tilde{u} \leq \widetilde{w}$.

From Proposition 6, we know that the order relation $\leq$ is a partial order on the set of all $L R$ fuzzy numbers.

2.4. Fully Fuzzy Linear Programming with LR Fuzzy Parameters. In this paper, we will consider the following model; that is,

$$
\begin{array}{ll}
\min & z(\tilde{x})=\tilde{c} \otimes \tilde{x} \\
\text { s.t. } & \widetilde{A} \otimes \tilde{x} \leq \tilde{b} \\
& \tilde{x} \geq \widetilde{0},
\end{array}
$$

or

$$
\begin{array}{cc}
\min & z(\tilde{x})=\tilde{c}_{1} \otimes \tilde{x}_{1} \oplus \tilde{c}_{2} \otimes \tilde{x}_{2} \oplus \cdots \oplus \tilde{c}_{n} \otimes \tilde{x}_{n} \\
\text { s.t. } & \tilde{a}_{i 1} \otimes \tilde{x}_{1} \oplus \tilde{a}_{i 2} \otimes \tilde{x}_{2} \oplus \cdots \oplus \tilde{a}_{i n} \otimes \tilde{x}_{n} \leq \tilde{b}_{i}, \\
& i=1,2, \ldots, m, \\
& \tilde{x}_{j} \geq \tilde{0}, \quad j=1,2, \ldots, n,
\end{array}
$$

where $\tilde{c}=\left[\widetilde{c}_{j}\right]_{1 \times n}, \widetilde{b}=\left[\widetilde{b}_{i}\right]_{m \times 1}, \widetilde{A}=\left[\tilde{a}_{i j}\right]_{m \times n}$, and $\tilde{x}=\left[\tilde{x}_{j}\right]_{n \times 1}$ represent $L R$ fuzzy matrices and vectors and $\widetilde{c}_{j}, \widetilde{b}_{i}, \widetilde{a}_{i j}$, and $\widetilde{x}_{j}$ are $L R$ flat fuzzy numbers. The order relations for comparing the $L R$ flat fuzzy numbers both in the objective function and the constraint inequalities are as shown in Definition 4.

\section{Proposed Method}

Steps of the proposed method are given to solve problem (20) as follows. This method is applicable to minimization of FFLP problems, and the solution method of maximization problems is similar to that of minimization ones.

Step 1. If all the parameters $\widetilde{c}_{j}, \widetilde{b}_{i}, \widetilde{a}_{i j}, \widetilde{x}_{j}$ are represented by $L R$ flat fuzzy numbers $\left(c_{j 1}, c_{j 2}, \alpha_{c_{j}}, \beta_{c_{j}}\right)_{L R},\left(b_{i 1}, b_{i 2}, \alpha_{b_{i}}, \beta_{b_{i}}\right)_{L R}$, $\left(a_{i j 1}, a_{i j 2}, \alpha_{a_{i j}}, \beta_{a_{i j}}\right)_{L R}$, and $\left(x_{j 1}, x_{j 2}, \alpha_{x_{j}}, \beta_{x_{j}}\right)_{L R}$, then the FFLP (20) can be written as

$$
\begin{gathered}
\min \quad z(\tilde{x})=\sum_{j=1}^{n}\left(\left(c_{j 1}, c_{j 2}, \alpha_{c_{j}}, \beta_{c_{j}}\right)_{L R}\right. \\
\left.\otimes\left(x_{j 1}, x_{j 2}, \alpha_{x_{j}}, \beta_{x_{j}}\right)_{L R}\right) \\
\text { s.t. } \quad \sum_{j=1}^{n}\left(\left(a_{i j 1}, a_{i j 2}, \alpha_{a_{i j}}, \beta_{a_{i j}}\right)_{L R} \otimes\left(x_{j 1}, x_{j 2}, \alpha_{x_{j}}, \beta_{x_{j}}\right)_{L R}\right) \\
\leq\left(b_{i 1}, b_{i 2}, \alpha_{b_{i}}, \beta_{b_{i}}\right)_{L R}, \quad i=1,2, \ldots, m, \\
\left(x_{j 1}, x_{j 2}, \alpha_{x_{j}}, \beta_{x_{j}}\right)_{L R} \geq(0,0,0,0,)_{L R}, \\
\quad j=1,2, \ldots, n .
\end{gathered}
$$

Step 2. Calculate $\left(c_{j 1}, c_{j 2}, \alpha_{c_{j}}, \beta_{c_{j}}\right)_{L R} \otimes\left(x_{j 1}, x_{j 2}, \alpha_{x_{j}}, \beta_{x_{j}}\right)_{L R}$ and $\left(a_{i j 1}, a_{i j 2}, \alpha_{a_{i j}}, \beta_{a_{i j}}\right)_{L R} \otimes\left(x_{j 1}, x_{j 2}, \alpha_{x_{j}}, \beta_{x_{j}}\right)_{L R}$, respectively, and suppose that $\left(c_{j 1}, c_{j 2}, \alpha_{c_{j}}, \beta_{c_{j}}\right)_{L R} \otimes\left(x_{j 1}, x_{j 2}, \alpha_{x_{j}}, \beta_{x_{j}}\right)_{L R}=$ $\left(x_{j 1}^{\prime}, x_{j 2}^{\prime}, \alpha_{x_{j}}^{\prime}, \beta_{x_{j}}^{\prime}\right)_{L R}$ and $\left(a_{i j 1}, a_{i j 2}, \alpha_{a_{i j}}, \beta_{a_{i j}}\right)_{L R} \otimes\left(x_{j 1}, x_{j 2}, \alpha_{x_{j}}\right.$, $\left.\beta_{x_{j}}\right)_{L R}=\left(p_{i j}, q_{i j}, \gamma_{i j}, \delta_{i j}\right)_{L R}$; then the FFLP problem obtained in Step 1 can be written as

$$
\min z(\tilde{x})=\left(\sum_{j=1}^{n} x_{j 1}^{\prime}, \sum_{j=1}^{n} x_{j 2}^{\prime}, \sum_{j=1}^{n} \alpha_{x_{j}}^{\prime}, \sum_{j=1}^{n} \beta_{x_{j}}^{\prime}\right)_{L R}
$$

s.t. $\quad\left(\sum_{j=1}^{n} p_{i j}, \sum_{j=1}^{n} q_{i j}, \sum_{j=1}^{n} \gamma_{i j}, \sum_{j=1}^{n} \delta_{i j}\right)_{L R} \leq\left(b_{i 1}, b_{i 2}, \alpha_{b_{i}}, \beta_{b_{i}}\right)_{L R}$,

$$
i=1,2, \ldots, m \text {, }
$$$$
\left(x_{j 1}, x_{j 2}, \alpha_{x_{j}}, \beta_{x_{j}}\right)_{L R} \geq(0,0,0,0,)_{L R},
$$ 
Step 3. According to the order relation defined above, the problem obtained in Step 2 is equivalent to

$$
\begin{array}{ll}
\min & \sum_{j=1}^{n} x_{j 1}^{\prime} \\
& \sum_{j=1}^{n} x_{j 2}^{\prime} \\
& \sum_{j=1}^{n}\left(x_{j 1}^{\prime}-\alpha_{x_{j}}^{\prime}\right) \\
& \sum_{j=1}^{n}\left(x_{j 2}^{\prime}+\beta_{x_{j}}^{\prime}\right) \\
& \sum_{j=1}^{n} p_{i j} \leq b_{i 1}, \quad i=1,2, \ldots, m, \\
& \sum_{j=1}^{n} q_{i j} \leq b_{i 2}, \quad i=1,2, \ldots, m, \\
& \sum_{j=1}^{n}\left(p_{i j}-\gamma_{i j}\right) \leq\left(b_{i 1}-\alpha_{b_{i}}\right), \quad i=1,2, \ldots, m, \quad \beta_{x_{j}} \geq 0, \\
& \sum_{j=1}^{n}\left(q_{i j}+\delta_{i j}\right) \leq\left(b_{i 2}+\beta_{b_{i}}\right), \quad i=1,2, \ldots, m, \\
& x_{j 1}-\alpha_{x_{j}} \geq 0, \quad j=1,2, \ldots, n . \\
&
\end{array}
$$

We denote $X=\left(x_{11}, x_{12}, \alpha_{x_{1}}, \beta_{x_{1}}, x_{21}, x_{22}, \alpha_{x_{2}}, \beta_{x_{2}}, \ldots\right.$, $\left.x_{n 1}, x_{n 2}, \alpha_{x_{n}}, \beta_{x_{n}}\right)^{T}, z_{1}(X)=\sum_{j=1}^{n} x_{j 1}^{\prime}, z_{2}(X)=\sum_{j=1}^{n} x_{j 2}^{\prime}$, $z_{3}(X)=\sum_{j=1}^{n}\left(x_{j 1}^{\prime}-\alpha_{x_{j}}^{\prime}\right), z_{4}(X)=\sum_{j=1}^{n}\left(x_{j 2}^{\prime}+\beta_{x_{j}}^{\prime}\right)$ and $D=\{X \mid X$ satisfies the constraints of programming (23) $\}$. Programming (23) may be written as the programming (24), below for short, as follows:

$$
\begin{array}{ll}
\min & z_{1}(X) \\
& z_{2}(X) \\
& z_{3}(X) \\
& z_{4}(X) \\
\text { s.t. } & X \in D .
\end{array}
$$

Obviously, programming (24) is a crisp multiobjective linear programming problem. In fact we have $z(\widetilde{x})=$ $\left(z_{1}(X), z_{2}(X), z_{1}(X)-z_{3}(X), z_{4}(X)-z_{2}(X)\right)$.

Step 4. Solve the subproblems

$$
\begin{array}{ll}
\min & z_{t}(X) \\
\text { s.t. } & X \in D,
\end{array}
$$

where $t=1,2,3,4$. We find optimal solutions $X_{1}, X_{2}, X_{3}$, and $X_{4}$, respectively. And the corresponding optimal values will be $z_{1}^{\min }=z_{1}\left(X_{1}\right), z_{2}^{\mathrm{min}}=z_{2}\left(X_{2}\right), z_{3}^{\mathrm{min}}=z_{3}\left(X_{3}\right)$, and $z_{4}^{\min }=z_{4}\left(X_{4}\right)$.

Step 5. Let $z_{t}^{\max }=\max \left\{z_{t}\left(X_{1}\right), z_{t}\left(X_{2}\right), z_{t}\left(X_{3}\right), z_{t}\left(X_{4}\right)\right\}, t=$ $1,2,3,4$, and the membership function of $z_{t}(X)$ is given by

$$
\mu_{z_{t}}\left(z_{t}(X)\right)= \begin{cases}1, & z_{t}(X)<z_{t}^{\min }, \\ \frac{z_{t}^{\max }-z_{t}(X)}{z_{t}^{\max }-z_{t}^{\min }}, & z_{t}^{\min } \leq z_{t}(X) \leq z_{t}^{\max }, \\ 0, & z_{t}(X)>z_{t}^{\max }\end{cases}
$$

where $t=1,2,3,4$.

Step 6. Let $I_{0}=\{1,2,3,4\}$; the MOLP problem obtained in Step 3 can be equivalently written as

$$
\begin{array}{ll}
\max & \lambda \\
\text { s.t. } & \mu_{z_{t}}\left(z_{t}(X)\right) \geq \lambda, \quad t \in I_{0}, \\
& X \in D .
\end{array}
$$

Suppose $X^{1}$ is one of the optimal solutions (if there exits only one optimal solution, $X^{1}$ is the unique one), and $\lambda^{1 *}$ is the optimal objective value (in fact, the optimal solution should be written as $\left(X^{1}, \lambda^{1 *}\right)$. Since $\lambda$ is an auxiliary variable, we denote $\left(X^{1}, \lambda^{1 *}\right)$ as $X^{1}$ for simplicity). Then $\mu_{z_{s_{1}}}\left(z_{s_{1}}\left(X^{1}\right)\right)=$ $\lambda^{1 *}$ for at least one $s_{1}$ in $I_{0}$. ( $s_{1}$ is an arbitrary element in the set $\left.J=\left\{j \mid \mu_{z_{j}}\left(z_{j}\left(X^{1}\right)\right)=\lambda^{1 *}\right\}\right)$.

Step 7. Let $I_{1}=I_{0}-\left\{s_{1}\right\}$, and solve the following crisp programming:

$$
\begin{array}{ll}
\max & \lambda \\
\text { s.t. } & \mu_{z_{t}}\left(z_{t}(X)\right) \geq \lambda, \quad t \in I_{1}, \\
& \mu_{z_{s_{1}}}\left(z_{s_{1}}(X)\right)=\lambda^{1 *}, \\
& X \in D .
\end{array}
$$

If $X^{2}$ is one of the optimal solutions and $\lambda^{2 *}$ is the optimal objective value, then $\mu_{z_{s_{2}}}\left(z_{s_{2}}\left(X^{2}\right)\right)=\lambda^{2 *}$ for at least one $s_{2}$ in $I_{1}$.

Step 8. Let $I_{2}=I_{0}-\left\{s_{1}, s_{2}\right\}$, and solve the following crisp programming:

$$
\begin{array}{ll}
\max & \lambda \\
\text { s.t. } & \mu_{z_{t}}\left(z_{t}(X)\right) \geq \lambda, \quad t \in I_{2}, \\
& \mu_{z_{s_{1}}}\left(z_{s_{1}}(X)\right)=\lambda^{1 *}, \\
& \mu_{z_{s_{2}}}\left(z_{s_{2}}(X)\right)=\lambda^{2 *}, \\
& X \in D .
\end{array}
$$


Suppose $X^{3}$ is one of the optimal solutions and $\lambda^{3 *}$ is the optimal objective value. Then $\mu_{z_{s_{3}}}\left(z_{s_{3}}\left(X^{3}\right)\right)=\lambda^{3 *}$ for at least one $s_{3}$ in $I_{2}$.

Step 9. Let $I_{3}=I_{0}-\left\{s_{1}, s_{2}, s_{3}\right\}$, and solve the following crisp programming:

$$
\begin{array}{ll}
\max & \lambda \\
\text { s.t. } & \mu_{z_{t}}\left(z_{t}(X)\right) \geq \lambda, \quad t \in I_{3}, \\
& \mu_{z_{s_{1}}}\left(z_{s_{1}}(X)\right)=\lambda^{1 *}, \\
& \mu_{s_{s_{2}}}\left(z_{s_{2}}(X)\right)=\lambda^{2 *}, \\
& \mu_{s_{s_{3}}}\left(z_{s_{3}}(X)\right)=\lambda^{3 *} \\
& X \in D
\end{array}
$$

Suppose $X^{4}$ is one of the optimal solutions, and $\lambda^{4 *}$ is the optimal objective value. Then $\mu_{z_{s_{4}}}\left(z_{s_{4}}\left(X^{4}\right)\right)=\lambda^{4 *}$ with $s_{4}$ in $I_{3}$.

Step 10. Take $X^{*}=X^{4}$ as the compromised optimal solution to programming (23) and generate the compromised optimal solution $\tilde{x}^{*}$ to programming (21) by $X^{*}$. Assuming

$$
\begin{gathered}
X^{*}=\left(x_{11}^{*}, x_{12}^{*}, \alpha_{x_{1}}^{*}, \beta_{x_{1}}^{*}, x_{21}, x_{22}^{*}, \alpha_{x_{2}}^{*},\right. \\
\left.\beta_{x_{2}}^{*}, \ldots, x_{n 1}, x_{n 2}^{*}, \alpha_{x_{n}}^{*}, \beta_{x_{n}}^{*}\right)^{T},
\end{gathered}
$$

we may obtain

$$
\begin{aligned}
\tilde{x}^{*}= & \left(\tilde{x}_{1}^{*}, \tilde{x}_{2}^{*}, \ldots, \tilde{x}_{n}^{*}\right)^{T} \\
= & \left(\left(x_{11}^{*}, x_{12}^{*}, \alpha_{x_{1}}^{*}, \beta_{x_{1}}^{*}\right)_{L R},\left(x_{21}^{*}, x_{22}^{*}, \alpha_{x_{2}}^{*}, \beta_{x_{2}}^{*}\right)_{L R},\right. \\
& \left.\ldots,\left(x_{n 1}^{*}, x_{n 2}^{*}, \alpha_{x_{n}}^{*}, \beta_{x_{n}}^{*}\right)_{L R}\right)^{T},
\end{aligned}
$$

and the corresponding objective value $z^{*}=z\left(\tilde{x}^{*}\right)$.

Remark $7\left(\left\{s_{1}, s_{2}, s_{3}, s_{4}\right\}=I=\{1,2,3,4\}\right)$. Some properties of the solutions obtained in Steps 6-10 are shown in the following proposition.

Proposition 8. Suppose $\mu_{z_{s_{j}}}\left(z_{s_{j}}\left(X^{*}\right)\right), X^{j}, \lambda^{j *}(j=1,2,3,4)$, and $X^{*}$ are the notations described in Steps 1-10; then

$$
\text { (1) } \begin{aligned}
\mu_{z_{1}} & \left(z_{s_{1}}\left(X^{*}\right)\right)=\lambda^{1 *}=\mu_{z_{s_{1}}}\left(z_{s_{1}}\left(X^{1}\right)\right), \\
\mu_{z_{s_{2}}}\left(z_{s_{2}}\left(X^{*}\right)\right) & =\lambda^{2 *}=\mu_{z_{s_{2}}}\left(z_{s_{2}}\left(X^{2}\right)\right), \\
\mu_{z_{s_{3}}}\left(z_{s_{3}}\left(X^{*}\right)\right) & =\lambda^{3 *}=\mu_{z_{s_{3}}}\left(z_{s_{3}}\left(X^{3}\right)\right), \\
\mu_{s_{s_{4}}}\left(z_{s_{4}}\left(X^{*}\right)\right) & =\lambda^{4 *}=\mu_{z_{s_{4}}}\left(z_{s_{4}}\left(X^{4}\right)\right),
\end{aligned}
$$

(2) $\lambda^{1 *} \leq \lambda^{2 *} \leq \lambda^{3 *} \leq \lambda^{4 *}$.
Proof. (1) From the results of Steps 6-9, it is obviously clear that

$$
\begin{aligned}
& \lambda^{1 *}=\mu_{z_{s_{1}}}\left(z_{s_{1}}\left(X^{1}\right)\right), \\
& \lambda^{2 *}=\mu_{z_{s_{2}}}\left(z_{s_{2}}\left(X^{2}\right)\right), \\
& \lambda^{3 *}=\mu_{z_{s_{3}}}\left(z_{s_{3}}\left(X^{3}\right)\right), \\
& \lambda^{4 *}=\mu_{z_{s_{4}}}\left(z_{s_{4}}\left(X^{4}\right)\right),
\end{aligned}
$$

and $\mu_{z_{s_{4}}}\left(z_{s_{4}}\left(X^{*}\right)\right)=\lambda^{4 *}$ with $X^{*}=X^{4}$. Since $X^{*}=X^{4}$ is an optimal solution to programming (30), we know that $X^{*}$ satisfies the constraints of programming (30), and so $\mu_{z_{s_{1}}}\left(z_{s_{1}}\left(X^{*}\right)\right)=\lambda^{1 *}, \mu_{z_{s_{2}}}\left(z_{s_{2}}\left(X^{*}\right)\right)=\lambda^{2 *}$, and $\mu_{z_{s_{3}}}\left(z_{s_{3}}\left(X^{*}\right)\right)=$ $\lambda^{3 *}$.

(2) In fact, $\left(X^{1}, \lambda^{1 *}\right)$ is an optimal solution to programming (27); therefore, it is a feasible solution. We have

$$
\begin{gathered}
\mu_{z_{t}}\left(z_{t}\left(X^{1}\right)\right) \geq \lambda^{1 *}, \quad t \in I_{1} \subseteq I_{0} \\
X^{1} \in D
\end{gathered}
$$

and it is obvious that $\mu_{z_{s_{1}}}\left(z_{s_{1}}\left(X^{*}\right)\right)=\lambda^{1 *}$ from the result of Step 6 . Hence, $\left(X^{1}, \lambda^{1 *}\right)$ is a feasible solution to programming (28). The objective value of $\left(X^{1}, \lambda^{1 *}\right)$ is $\lambda^{1 *}$, and the optimal objective value of programming (28) is $\lambda^{2 *}$; so we get $\lambda^{1 *} \leq$ $\lambda^{2 *}$. It is similar to prove $\lambda^{2 *} \leq \lambda^{3 *}$ and $\lambda^{3 *} \leq \lambda^{4 *}$.

\section{Numerical Example}

In this section, we present a numerical example to illustrate the feasibility of the solution method proposed in Section 3.

We aim to find the compromised optimal solution and corresponding objective value of the following fully fuzzy linear programming problem:

$$
\begin{aligned}
& \max z(\tilde{x})=z\left(\tilde{x}_{1}, \tilde{x}_{2}\right) \\
& =(6,7,1,2)_{L R} \otimes \tilde{x}_{1} \oplus(7,9,1,2)_{L R} \otimes \tilde{x}_{2} \\
& \text { s.t. } \quad(9,10,2,1)_{L R} \otimes \tilde{x}_{1} \oplus(1,1,1,1)_{L R} \otimes \tilde{x}_{2} \\
& \leq(50,55,4,3)_{L R}, \\
& (2,3,1,1)_{L R} \otimes \tilde{x}_{1} \oplus(4,5,1,2)_{L R} \otimes \tilde{x}_{2} \\
& \leq(66,70,3,5)_{L R}, \\
& \tilde{x}_{1} \geq \tilde{0}, \quad \tilde{x}_{2} \geq \tilde{0},
\end{aligned}
$$

where $\tilde{x}_{1}=\left(x_{11}, x_{12}, \alpha_{1}, \beta_{1}\right)_{L R}$ and $\tilde{x}_{2}=\left(x_{21}, x_{22}, \alpha_{2}, \beta_{2}\right)_{L R}$. 
TABLE 1: The optimal values and solutions of the four subproblems.

\begin{tabular}{lll}
\hline & The optimal objective value & The optimal solution \\
\hline (a) & $z_{1}^{\max }=70$ & $X_{1}=(0,0.3223,0,0.2887,10,10.1163,7.0903,0.1898)^{T}$ \\
(b) & $z_{2}^{\max }=103.3188$ & $X_{2}=(1.6015,3.7101,1.6039,0,4.2500,8.5942,3.1084,0)^{T}$ \\
(c) & $z_{3}^{\max }=50$ & $X_{3}=(0,0.2544,0,0.2973,10,10.0893,0,0.2179)^{T}$ \\
(d) & $z_{4}^{\max }=142.5882$ & $X_{4}=(1.4272,4.2157,1.2516,0,0,0,0,11.6275)^{T}$ \\
\hline
\end{tabular}

According to Steps 1 and 2 in the proposed method, we obtain the following programming:

$$
\begin{aligned}
& \max z=\left(6 x_{11}+7 x_{21}, 7 x_{12}+9 x_{22}, 6 \alpha_{1}+7 \alpha_{2}\right. \\
& \left.+x_{11}+2 x_{21}, 7 \beta_{1}+9 \beta_{2}+2 x_{12}+x_{22}\right)_{L R} \\
& \text { s.t. } \quad\left(9 x_{11}+x_{21}, 10 x_{12}+x_{22}, 9 \alpha_{1}+\alpha_{2}+2 x_{11}\right. \\
& \left.+x_{21}, 10 \beta_{1}+\beta_{2}+x_{12}+x_{22}\right)_{L R} \\
& \leq(50,55,4,3)_{L R}, \\
& \left(2 x_{11}+4 x_{21}, 3 x_{12}+5 x_{22}, 2 \alpha_{1}+4 \alpha_{2}+x_{11}\right. \\
& \left.+x_{21}, 3 \beta_{1}+5 \beta_{2}+x_{12}+2 x_{22}\right)_{L R} \\
& \leq(66,70,3,5)_{L R}, \\
& \left(x_{11}, x_{12}, \alpha_{1}, \beta_{1}\right)_{L R} \geq(0,0,0,0)_{L R}, \\
& \left(x_{21}, x_{22}, \alpha_{2}, \beta_{2}\right)_{L R} \geq(0,0,0,0)_{L R} \text {. }
\end{aligned}
$$

By Step 3, the programming above is transformed into the following programming:

$$
\begin{array}{ll}
\max & z_{1}=6 x_{11}+7 x_{21} \\
& z_{2}=7 x_{12}+9 x_{22} \\
& z_{3}=5 x_{11}+5 x_{21}-6 \alpha_{1}-7 \alpha_{2} \\
& z_{4}=9 x_{12}+10 x_{22}+7 \beta_{1}+9 \beta_{2} \\
\text { s.t. } \quad & 9 x_{11}+x_{21} \leq 50, \\
& 10 x_{12}+x_{22} \leq 55, \\
& 7 x_{11}-9 \alpha_{1}-\alpha_{2} \leq 46, \\
& 11 x_{12}+2 x_{22}+10 \beta_{1}+\beta_{2} \leq 58, \\
& 2 x_{11}+4 x_{21} \leq 66, \\
& 3 x_{12}+5 x_{22} \leq 70, \\
& x_{11}+3 x_{21}-2 \alpha_{1}-4 \alpha_{2} \leq 63, \\
& 4 x_{12}+7 x_{22}+3 \beta_{1}+5 \beta_{2} \leq 75, \\
& x_{11}-\alpha_{1} \geq 0, \quad x_{21}-\alpha \geq 0, \quad x_{21} \leq x_{22}, \\
& x_{11} \leq x_{12}, \quad \beta_{1}, \beta_{2} \geq 0 . \\
& \alpha_{1}, \alpha_{2}, \beta \beta_{1},
\end{array}
$$

Programming (38) can be abbreviated to the following programming:

$$
\begin{array}{ll}
\max & z_{1}(X) \\
& z_{2}(X) \\
& z_{3}(X) \\
& z_{4}(X) \\
\text { s.t. } & X \in D,
\end{array}
$$

where $X=\left(x_{11}, x_{12}, \alpha_{1}, \beta_{1}, x_{21}, x_{22}, \alpha_{2}, \beta_{2}\right)^{T}$.

Solve the following subproblems:

$$
\begin{aligned}
& \text { (a) } \max z_{1}(X) \\
& \text { s.t. } X \in D \text {, } \\
& \text { (b) } \max z_{2}(X) \\
& \text { s.t. } X \in D \text {, } \\
& \text { s.t. } X \in D \text {, } \\
& \text { (d) } \max z_{4}(X) \\
& \text { s.t. } X \in D \text {, }
\end{aligned}
$$

respectively, and we obtain the optimal objective value and one of the optimal solutions as shown in Table 1.

According to $z_{t}^{\min }=\min \left\{z_{t}\left(X_{1}\right), z_{t}\left(X_{2}\right), z_{t}\left(X_{3}\right), z_{t}\left(X_{4}\right)\right\}$, we acquire the lower objective values $z_{1}^{\min }=8.5631, z_{2}^{\min }=$ 29.5098, $z_{3}^{\min }=0.3681$, and $z_{4}^{\min }=107.2245$, with corresponding membership functions given below. Consider

$$
\mu_{z_{1}}\left(z_{1}(X)\right)=\left\{\begin{array}{l}
1, \\
z_{1}(X)>70 ; \\
\frac{z_{1}(X)-8.5631}{70-8.5631}, \\
8.5631 \leq z_{1}(X) \leq 70 ; \\
0, \quad \\
z_{1}(X)<8.5631,
\end{array}\right.
$$




$$
\begin{aligned}
& \mu_{z_{2}}\left(z_{2}(X)\right)=\left\{\begin{array}{l}
1, \\
z_{2}(X)>103.3188 \\
\frac{z_{2}(X)-29.5098}{103.3188-29.5098} \\
29.5098 \leq z_{2}(X) \leq 103.3188 ; \\
0, \\
z_{2}(X)<29.5098
\end{array}\right. \\
& \mu_{z_{3}}\left(z_{3}(X)\right)=\left\{\begin{array}{l}
1, \\
z_{3}(X)>50 \\
\frac{z_{3}(X)-0.3681}{50-0.3681} \\
0.3681 \leq z_{3}(X) \leq 50 \\
0, \\
z_{3}(X)<0.3681
\end{array}\right. \\
& \mu_{z_{4}}\left(z_{4}(X)\right)=\left\{\begin{array}{l}
1, \\
z_{4}(X)>142.5882 ; \\
\frac{z_{4}(X)-107.2245}{142.5882-107.2245} \\
107.2245 \leq z_{4}(X) \leq 142.5882 ; \\
0, \quad \\
z_{4}(X)<107.2245 .
\end{array}\right.
\end{aligned}
$$

By Steps 4-6, we get

$\max \lambda$

$$
\begin{array}{ll}
\text { s.t. } \quad & \mu_{z_{1}}\left(z_{1}(X)\right)=\frac{6 x_{11}+7 x_{21}-8.5631}{70-8.5631} \geq \lambda, \\
& \mu_{z_{2}}\left(z_{2}(X)\right)=\frac{7 x_{12}+9 x_{22}-29.5098}{103.3188-29.5098} \geq \lambda, \\
& \mu_{z_{3}}\left(z_{3}(X)\right) \\
= & \frac{5 x_{11}+5 x_{21}-6 \alpha_{1}-7 \alpha_{2}-0.3681}{50-0.3681} \geq \lambda, \\
& \mu_{z_{4}}\left(z_{4}(X)\right) \\
= & \frac{9 x_{12}+10 x_{22}+7 \beta_{1}+9 \beta_{2}-107.2245}{142.5882-107.2245} \geq \lambda,
\end{array}
$$

$X \in D$.

The optimal objective value is $\lambda^{1 *}=0.6033$, and one of the optimal solutions is $X^{1}=(2.5901,3.9107,0.4927,0$, 4.7997, 5.1848, 0.3109, 4.6127) ${ }^{T}$.

Calculate the value of the membership function of $z_{t}(X)$ $(t=1,2,3,4)$ at $X=X^{1}$, and we get $\mu_{z_{1}}\left(z_{1}\left(X^{1}\right)\right)=$ $0.6604, \mu_{z_{2}}\left(z_{2}\left(X^{1}\right)\right)=0.6033, \mu_{z_{3}}\left(z_{3}\left(X^{1}\right)\right)=0.6336$, and $\mu_{z_{4}}\left(z_{4}\left(X^{1}\right)\right)=0.6033$.
Solve the following problem:

$$
\begin{array}{ll}
\max & \lambda \\
\text { s.t. } & \mu_{z_{1}}\left(z_{1}(X)\right)=\frac{6 x_{11}+7 x_{21}-8.5631}{70-8.5631} \geq \lambda, \\
& \mu_{z_{2}}\left(z_{2}(X)\right)=\frac{7 x_{12}+9 x_{22}-29.5098}{103.3188-29.5098} \geq \lambda, \\
& \mu_{z_{3}}\left(z_{3}(X)\right) \\
& =\frac{5 x_{11}+5 x_{21}-6 \alpha_{1}-7 \alpha_{2}-0.3681}{50-0.3681} \geq \lambda, \\
& \mu_{z_{4}}\left(z_{4}(X)\right) \\
& =\frac{9 x_{12}+10 x_{22}+7 \beta_{1}+9 \beta_{2}-107.2245}{142.5882-107.2245}=0.6033
\end{array}
$$$$
X \in D \text {. }
$$

The optimal objective value is $\lambda^{2 *}=0.6033$, and one of the optimal solutions is $X^{2}=(2.8,3.9107,0.3135,0,4.7161$, $5.1850,0.4310,4.6124)^{T}$.

Calculate the value of the membership function of $z_{t}(X)$ $(t=1,2,3,4)$ at $X=X^{2}$, and we get $\mu_{z_{1}}\left(z_{1}\left(X^{2}\right)\right)=$ $0.6714, \mu_{z_{2}}\left(z_{2}\left(X^{2}\right)\right)=0.6033, \mu_{z_{3}}\left(z_{3}\left(X^{2}\right)\right)=0.6511$, and $\mu_{z_{4}}\left(z_{4}\left(X^{2}\right)\right)=0.6033$.

Solve the following problem:

$\max \lambda$

$$
\begin{array}{ll}
\text { s.t. } \quad & \mu_{z_{1}}\left(z_{1}(X)\right)=\frac{6 x_{11}+7 x_{21}-8.5631}{70-8.5631} \geq \lambda, \\
& \mu_{z_{3}}\left(z_{3}(X)\right) \\
& =\frac{5 x_{11}+5 x_{21}-6 \alpha_{1}-7 \alpha_{2}-0.3681}{50-0.3681} \geq \lambda, \\
& \mu_{z_{2}}\left(z_{2}(X)\right)=\frac{7 x_{12}+9 x_{22}-29.5098}{103.3188-29.5098}=0.6033 \\
& \mu_{z_{4}}\left(z_{4}(X)\right) \\
= & \frac{9 x_{12}+10 x_{22}+7 \beta_{1}+9 \beta_{2}-107.2245}{142.5882-107.2245}=0.6033
\end{array}
$$

$X \in D$.

The optimal objective value is $\lambda^{3 *}=0.7126$, and one of the optimal solutions is $X^{3}=(2.6750,3.9107,0.1158,0.0005$, $5.1850,5.1850,0.2608,4.6125)^{T}$.

Calculate the value of the membership function of $z_{t}(X)(t=1,2,3,4)$ at $X=X^{3}$, and we get $\mu_{z_{1}}\left(z_{1}\left(X^{3}\right)\right)=$ $0.7126, \mu_{z_{2}}\left(z_{2}\left(X^{3}\right)\right)=0.6033, \mu_{z_{3}}\left(z_{3}\left(X^{3}\right)\right)=0.7274$, and $\mu_{z_{4}}\left(z_{4}\left(X^{3}\right)\right)=0.6033$. 
TABLE 2: Values of the four membership functions at $X^{j}$

\begin{tabular}{lcccc}
\hline & $\mu_{z_{1}}\left(z_{1}(X)\right)$ & $\mu_{z_{2}}\left(z_{2}(X)\right)$ & $\mu_{z_{3}}\left(z_{3}(X)\right)$ & 0.6336 \\
\hline$X=X^{1}$ & 0.6604 & 0.6033 & $\mu_{z_{4}}\left(z_{4}(X)\right)$ \\
$X=X^{2}$ & 0.6714 & 0.6033 & 0.6511 & 0.6033 \\
$X=X^{3}$ & 0.7126 & 0.6033 & 0.7274 & 0.6033 \\
$X=X^{4}$ & 0.7126 & 0.6033 & 0.7844 & 0.6033 \\
\hline
\end{tabular}

TABLE 3: Values of the objective function $z(\widetilde{x})$ at $\tilde{x}^{j}$.

\begin{tabular}{lc}
\hline & $z(\tilde{x})$ \\
\hline$\tilde{x}=\tilde{x}^{1}$ & $(49.13,74.03,17.32,38.96)_{L R}$ \\
$\tilde{x}=\tilde{x}^{2}$ & $(49.81,74.03,17.13,38.96)_{L R}$ \\
$\tilde{x}=\tilde{x}^{3}$ & $(52.34,74.03,15.56,38.96)_{L R}$ \\
$\tilde{x}=\tilde{x}^{4}$ & $(52.34,74.03,13.04,38.96)_{L R}$ \\
\hline
\end{tabular}

Solve the following problem:

$\max \lambda$

$$
\begin{aligned}
& \text { s.t. } \quad \mu_{z_{3}}\left(z_{3}(X)\right)=\frac{5 x_{11}+5 x_{21}-6 \alpha_{1}-7 \alpha_{2}-0.3681}{50-0.3681} \geq \lambda, \\
& \mu_{z_{1}}\left(z_{1}(X)\right)=\frac{6 x_{11}+7 x_{21}-8.5631}{70-8.5631}=0.7126, \\
& \mu_{z_{2}}\left(z_{2}(X)\right)=\frac{7 x_{12}+9 x_{22}-29.5098}{103.3188-29.5098}=0.6033, \\
& \mu_{z_{4}}\left(z_{4}(X)\right) \\
& =\frac{9 x_{12}+10 x_{22}+7 \beta_{1}+9 \beta_{2}-107.2245}{142.5882-107.2245}=0.6033,
\end{aligned}
$$

$X \in D$

The optimal objective value is $\lambda^{4 *}=0.7844$, and one of the optimal solutions is $X^{4}=(2.6754,3.9106,0,0,5.1843$, 5.1848, 0, 4.6128) $)^{T}$.

Calculate the value of the membership function of $z_{t}(X)$ $(t=1,2,3,4)$ at $X=X^{4}$, and we get $\mu_{z_{1}}\left(z_{1}\left(X^{4}\right)\right)=$ $0.7126, \mu_{z_{2}}\left(z_{2}\left(X^{4}\right)\right)=0.6033, \mu_{z_{3}}\left(z_{3}\left(X^{4}\right)\right)=0.7844$, and $\mu_{z_{4}}\left(z_{4}\left(X^{4}\right)\right)=0.6033$.

Generate $\tilde{x}^{j}$ by $X^{j}(j=1,2,3,4)$, following Step 10, and calculate the value of $z\left(\tilde{x}^{j}\right)$. As shown in Tables 2 and 3 , the solution $\tilde{x}^{j+1}\left(\right.$ or $\left.X^{j+1}\right)$ is better than $\tilde{x}^{j}\left(\right.$ or $\left.X^{j}\right), j=1,2,3$.

Following Step 10, we find

$$
X^{*}=X^{4}=(2.6754,3.9106,0,0,5.1843,5.1848,0,4.6128)^{T} \text {. }
$$

Therefore,

$$
\begin{aligned}
\tilde{x}^{*}= & \tilde{x}^{4} \\
= & \left((2.6754,3.9106,0,0)_{L R},\right. \\
& \left.\quad(5.1843,5.1848,0,4.6128)_{L R}\right)^{T},
\end{aligned}
$$

serves as the compromised optimal solution with corresponding objective value

$$
z^{*}=z\left(\tilde{x}^{*}\right)=(52.34,74.03,13.04,38.96)_{L R} .
$$

\section{Concluding Remarks}

To the end, we show the following concluding remarks.

(1) In this paper, we proposed a new method to find the compromised optimal solution to the fully fuzzy linear programming problems with all the parameters and variables represented by $L R$ flat fuzzy numbers. The solution is also an $L R$ flat fuzzy number. In this sense, we get an exact solution, which may give more help to the decision makers.

(2) The order relation in the objective function is the same as that in the constraint inequalities. Based on the definition of the order relation, the FFLP can be equivalently transformed into a MOLP, which is a crisp programming that is easy to be solved.

(3) Considering the MOLP problem, classical fuzzy programming method is modified for obtaining the compromised optimal solution.

\section{Conflict of Interests}

The authors declare that there is no conflict of interests regarding the publication of this paper.

\section{Acknowledgments}

The authors would like to thank the editor and the anonymous reviewers for their valuable comments, which have been very helpful in improving the paper. This work is supported by the PhD Start-up Fund of Natural Science Foundation of Guangdong Province, China (S2013040012506), and the China Postdoctoral Science Foundation Funded Project (2014M562152).

\section{References}

[1] R. E. Bellman and L. A. Zadeh, "Decision-making in a fuzzy environment," Management Science, vol. 17, pp. B141-B164, 1970.

[2] M. Dehghan, B. Hashemi, and M. Ghatee, "Computational methods for solving fully fuzzy linear systems," Applied Mathematics and Computation, vol. 179, no. 1, pp. 328-343, 2006.

[3] M. Dehghan and B. Hashemi, "Solution of the fully fuzzy linear systems using the decomposition procedure," Applied 
Mathematics and Computation, vol. 182, no. 2, pp. 1568-1580, 2006.

[4] M. Dehghan, B. Hashemi, and M. Ghatee, "Solution of the fully fuzzy linear systems using iterative techniques," Chaos, Solitons \& Fractals, vol. 34, no. 2, pp. 316-336, 2007.

[5] A. Kumar, J. Kaur, and P. Singh, "A new method for solving fully fuzzy linear programming problems," Applied Mathematical Modelling, vol. 35, no. 2, pp. 817-823, 2011.

[6] F. Hosseinzadeh Lotfi, T. Allahviranloo, M. Alimardani Jondabeh, and L. Alizadeh, "Solving a full fuzzy linear programming using lexicography method and fuzzy approximate solution," Applied Mathematical Modelling, vol. 33, no. 7, pp. 3151-3156, 2009.

[7] T. Allahviranloo, F. H. Lotfi, M. K. Kiasary, N. A. Kiani, and L. Alizadeh, "Solving fully fuzzy linear programming problem by the ranking function," Applied Mathematical Sciences, vol. 2, no. 1-4, pp. 19-32, 2008.

[8] J. Kaur and A. Kumar, "Exact fuzzy optimal solution of fully fuzzy linear programming problems with unrestricted fuzzy variables," Applied Intelligence, vol. 37, no. 1, pp. 145-154, 2012.

[9] J. Kaur and A. Kumar, "Mehar's method for solving fully fuzzy linear programming problems with $L-R$ fuzzy parameters," Applied Mathematical Modelling, vol. 37, no. 12-13, pp. 71427153, 2013.

[10] J. J. Buckley and T. Feuring, "Evolutionary algorithm solution to fuzzy problems: fuzzy linear programming," Fuzzy Sets and Systems, vol. 109, no. 1, pp. 35-53, 2000.

[11] S. M. Hashemi, M. Modarres, E. Nasrabadi, and M. M. Nasrabadi, "Fully fuzzified linear programming, solution and duality," Journal of Intelligent and Fuzzy Systems, vol. 17, no. 3, pp. 253-261, 2006.

[12] Y. R. Fan, G. H. Huang, and A. L. Yang, "Generalized fuzzy linear programming for decision making under uncertainty: feasibility of fuzzy solutions and solving approach," Information Sciences, vol. 241, pp. 12-27, 2013.

[13] S. H. Nasseri and F. Zahmatkesh, "Huang method for solving fully fuzzy linear system of equations," Journal of Mathematics and Computer Science, vol. 1, pp. 1-5, 2010.

[14] H.-J. Zimmermann, "Fuzzy programming and linear programming with several objective functions," Fuzzy Sets and Systems, vol. 1, no. 1, pp. 45-55, 1978.

[15] B.-Y. Cao, Fuzzy Geometric Programming, Kluwer Academic Publishers, Boston, Mass, USA, 2002.

[16] D. Dubois and H. Prade, Fuzzy Sets and Systems, Theory and Applications, vol. 144 of Mathematics in Science and Engineering, Academic Press, New York, NY, USA, 1980. 


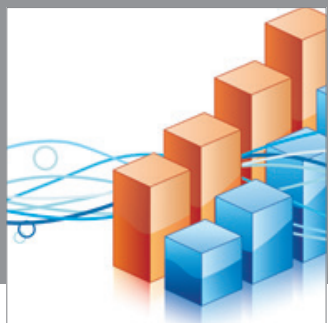

Advances in

Operations Research

mansans

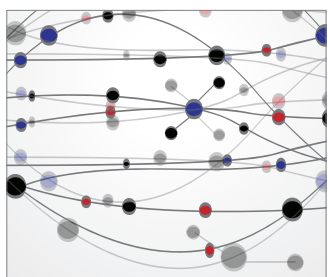

The Scientific World Journal
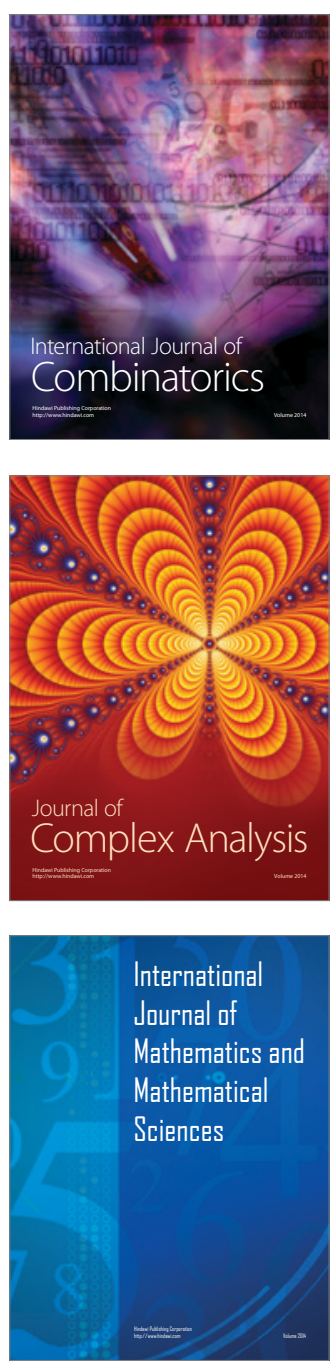
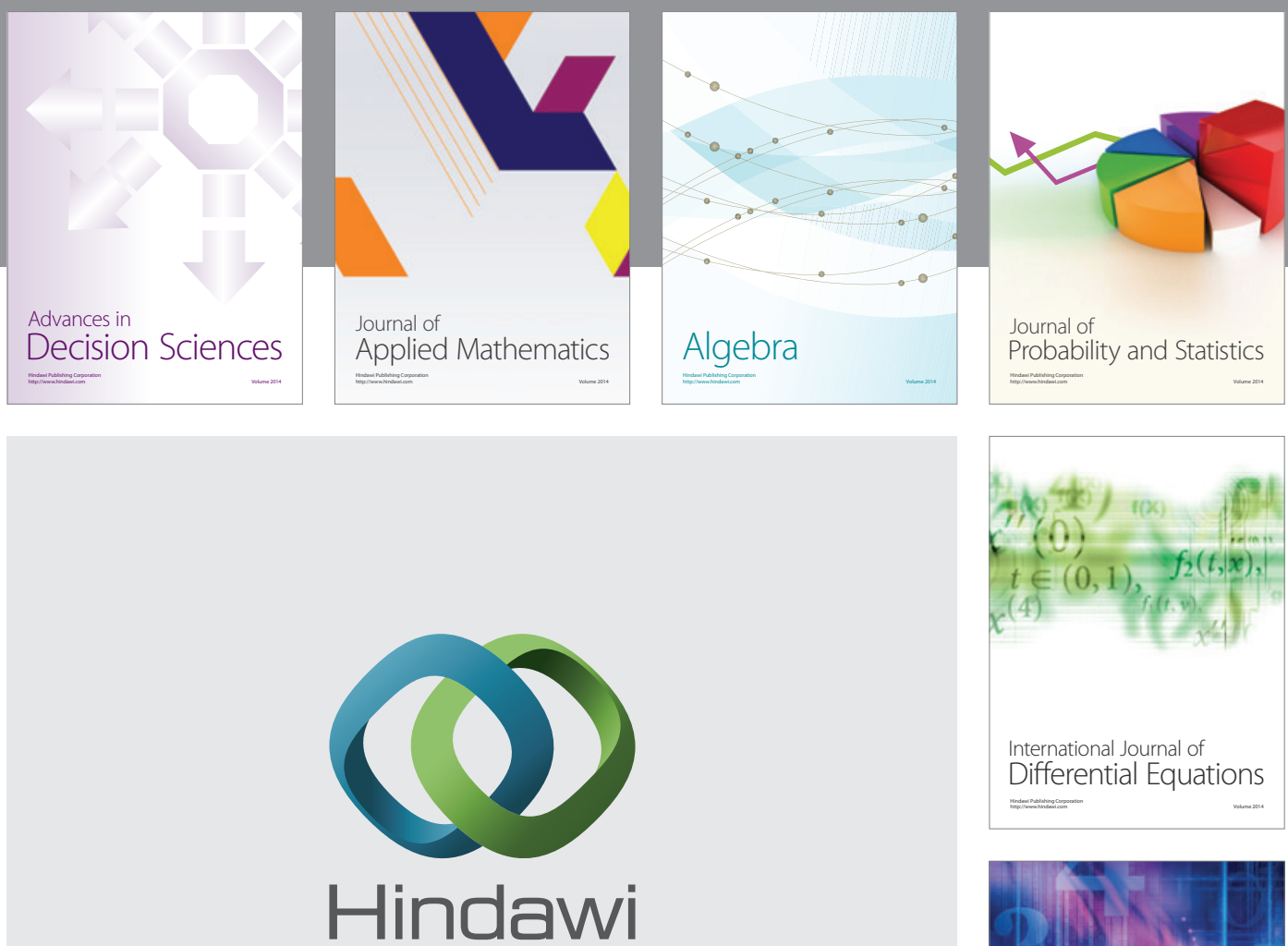

Submit your manuscripts at http://www.hindawi.com
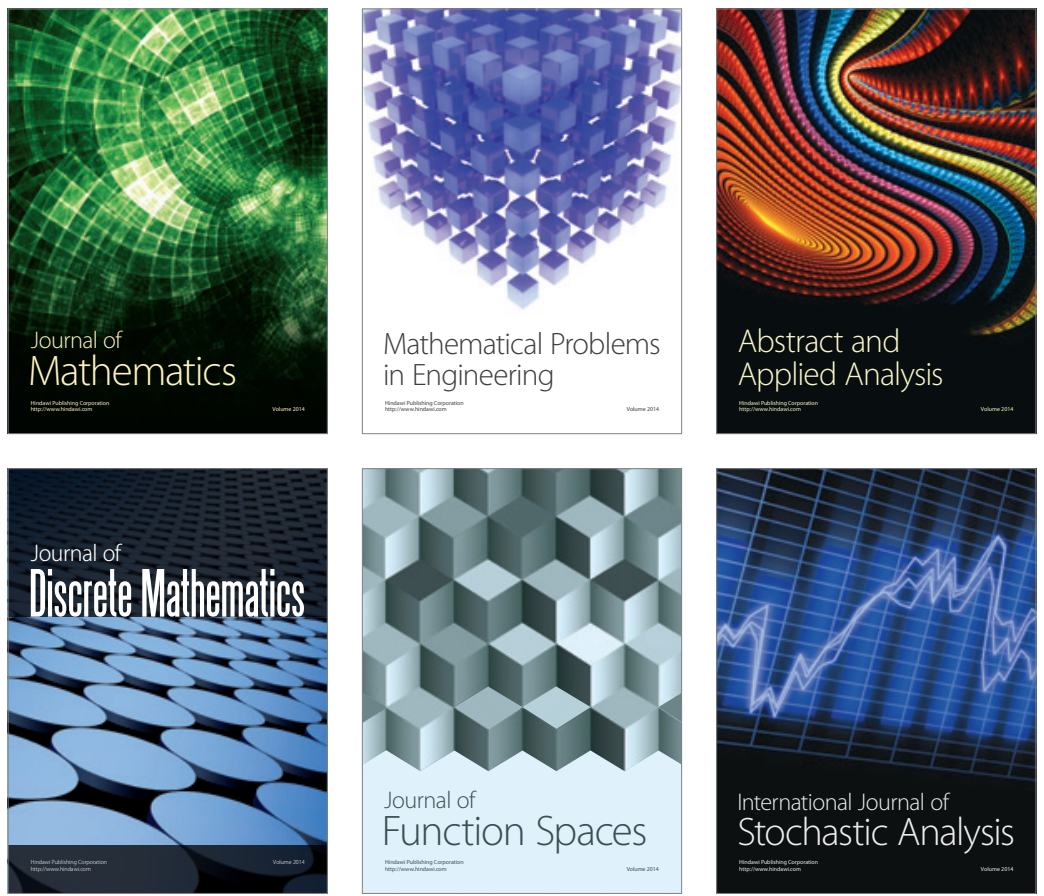

Journal of

Function Spaces

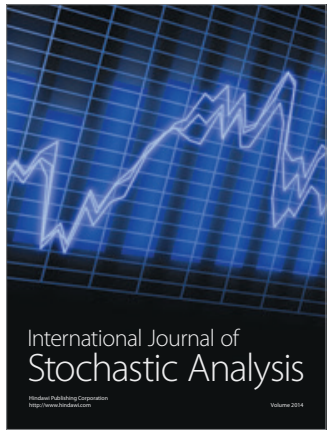

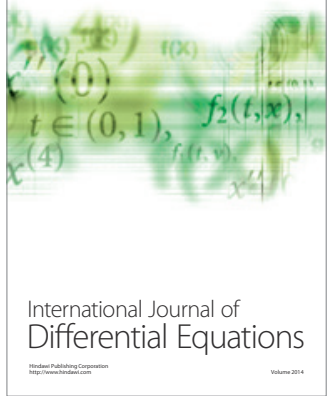
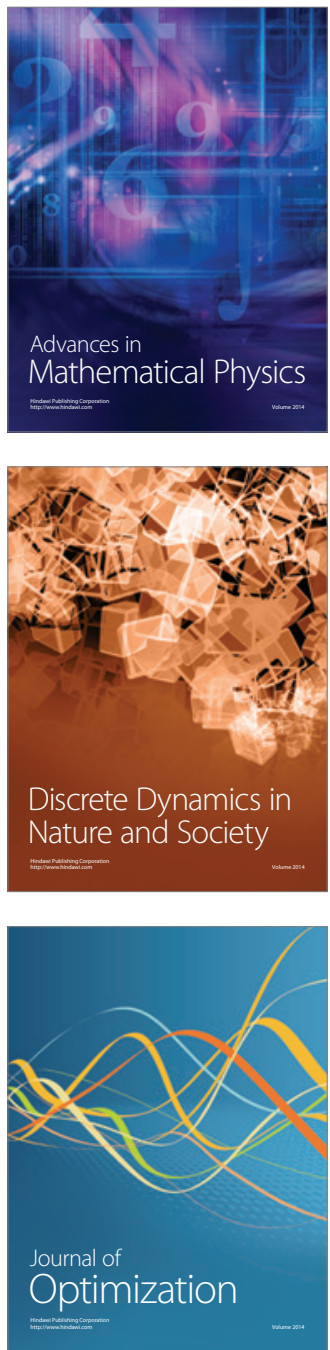